\title{
A Hierarchical Modeling Method for the Cladding Part
}

\author{
Yang Junru ${ }^{1, a}$, Huan Xiaoqian ${ }^{1}$, Zhang Lili ${ }^{1}$, Zhang Daozhong ${ }^{2}$,Zhang Yuekan ${ }^{1}$ \\ ${ }^{1}$ College of Mechanical \& Electronic Engineering, Shandong University of Science and Technology, \\ Qingdao 266590, China \\ ${ }^{2}$ Shandong Hoteam Software Co. Ltd.,Jinan 250101,China \\ ajryangzhang@163.com
}

Keywords: cladding part, hierarchical modeling method, bonding strength

\begin{abstract}
According to the material composition and structure characteristics, a hierarchical modeling method for the cladding part is proposed. The clad, interface and substrate are firstly modeled respectively, then they are glued together, meanwhile the bonding strength is considered. The cladding part modeling system prototype is designed based on the software SINOVATION.
\end{abstract}

\section{Introduction}

The cladding part is a new kind of layered composite part with excellent comprehensive properties and can be widely used in many fields of aerospace, automobile, machinery and so on. The part consists of three layers of the substrate, interface and clad, as shown in Fig.1. The substrate and clad are homogeneous materials, while the interface layer is the gradual material varying from the substrate to the clad. There have two methods to model the part. The first one ignores the interface and views the part as a physical combination of two homogeneous layers. The other one models the cladding part as a functionally graded material part, ignoring the bonding strength between layers. Both methods are not accurate to the actual structure and properties of cladding parts. A hierarchical modeling method for the part is proposed in the paper, in which, the clad, interface and substrate are firstly

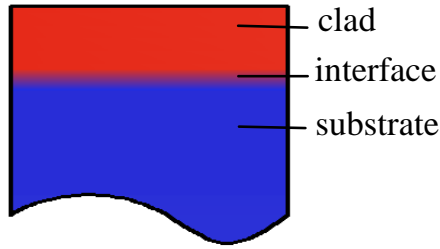

Fig. 1 The cladding part structure modeled respectively, then glue them together, meanwhile the bonding strength is considered.

\section{Hierarchical Modeling Method for the Cladding Part}

Five steps are contained in the hierarchical modeling method: models of the substrate, interface, clad, glue and assistant. The interface layer modeling includes basic and assistant modeling.

Substrate Layer Modeling. Substrate modeling mainly includes geometry modeling, material information addition and visualization. The geometry modeling and visualization methods are similar to those of homogeneous parts. Material feature is regarded as an additive attribution to the substrate layer.

Interface Layer Modeling. Interface modeling mainly includes geometry, material, visualization and assistant modeling.

Geometry Modeling of the Interface Layer. In the process of the interface geometry modeling, the geometric and topologic information of the substrate surface to be added an interface layer is firstly queried and extracted from the database. Using the obtained information, with the related copy algorithm, a new surface with the same shape, size, position and direction as the original surface on the substrate is generated, stretching the new surface, the interface geometry model is built.

Material Modeling of the Interface Layer. Existing heterogeneous material representation methods mainly include voxel based model, B-rep based model, explicit functional representation based model, compound model, etc[1-3]. The voxel based model method is adapted to model the interface functional graded material. 
The interface material modeling includes model voxelization and material composition calculation. Voxelization means dividing the geometry model into voxel unit grids (e.g. hexahedron and tetrahedron unit grids). All the grids are connected to each other at the nodes and with geometric continuity. Material composition calculation means calculating the material composition of every voxel central point to represent the voxel material composition. The whole heterogeneous part material attribution is presented by all the voxel material compositions as shown in Fig.2. The material composition of a voxel central point is obtained by distance function $f\left(d_{s}\right)$, where $d_{s}$ denotes the distance between the voxel central point and the gradient source $S$ ( the start surface of the interface). Gradient source is the material variation origin, and is also the reference of material distribution in the heterogeneous parts.

The following is the detailed interface material composition representation and calculation.

(1)Material information representation of each voxel. In Fig.2, the central point of a voxel in the interface layer is represented as $(\mathrm{P}, \mathrm{M}(\mathrm{P}))$, $\mathrm{P}$ denotes the central point geometric position expressed with $(\mathrm{x}, \mathrm{y}, \mathrm{z}), \mathrm{M}(\mathrm{P})$ is its material distribution accurately expressed with a two-dimension vector $\left[w_{s}, w_{e}\right]$, where $w_{s}$ and $w_{e}$ denote compositions of the start substrate material and the end clad material respectively. As the material composition at any point is made up of the substrate and clad materials, then, $w_{s}+w_{e}=1$. From the start to the end surface in interface layer, $w_{s}$ reduces from 1 to $0, w_{e}$ increases from 0 to 1 . For example, on the start surface, $\mathrm{M}(\mathrm{P})=[1,0]$, and on the end surface, $\mathrm{M}(\mathrm{P})=[0,1]$.

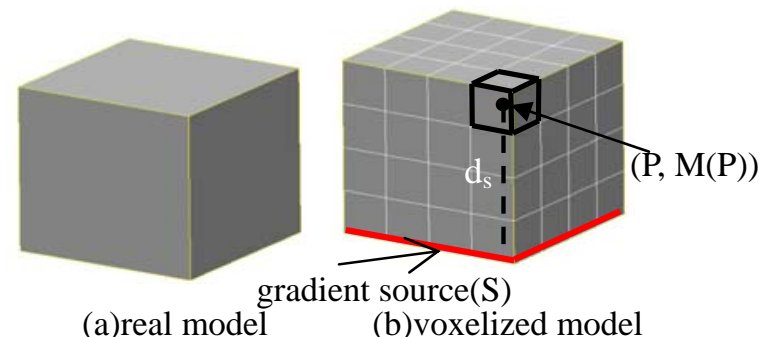

Fig.2 Model voxelization

(2)Material composition calculation of each voxel. The material composition calculation process of each voxel is described as follows. First, calculate the coordination of each voxel central point $\mathrm{P}$, and then calculate the minimum distance $d_{s}$ from the point $\mathrm{P}$ to the start surface in the interface layer, At last, the material composition of the point $\mathrm{P}$ can be obtained according to the distance function $f\left(d_{s}\right)$ which decides $w_{s}$ and $w_{e}$.

The formulas to calculate material composition of each voxel central point are as follows.

$$
\begin{aligned}
& M(P)=w_{s} M_{s}+w_{e} M_{e}=\left[w_{s}, w_{e}\right]\left[\begin{array}{l}
M_{s} \\
M_{e}
\end{array}\right],\left(0 \leq w_{s} \leq 1,0 \leq w_{e} \leq 1\right) \\
& d_{s}=\operatorname{dist}(P, S)
\end{aligned}
$$

Where, $M_{s}$ and $M_{e}$ denote the materials of the substrate and clad respectively.

The distance function $f\left(d_{s}\right)$ reflects the substrate material distribution rule in interface layer, it may be linear or nonlinear, which is related to the material category, thickness ratio of the substrate and clad, and the cladding part fabrication. Linear distribution is the easiest, it is as follows.

$$
w_{e}=1-w_{s}=1-f\left(d_{s}\right)=1-\frac{d_{s}}{h}
$$

Where $h$ is the interface layer thickness.

In the process of calculating material composition, $d_{s}$ in the function $f\left(d_{s}\right)$ is the minimum distance between an arbitrary point and the start surface in interface layer. Methods to calculate $d_{s}$ are grid algorithm[4], Nelder-Meade algorithm[5], particle swarm optimization algorithm[6], etc.

Visualization of the Interface Layer. Boundary visualization method [7] is used to visualize the interface layer material. The visualization process consists of four steps: boundary discretization, material composition calculation, color mapping and grid rendering.

(1)Boundary discretization: Each interface surface layer is discretized into the triangular grids. 
(2)Material composition calculation: After the boundary discretization, material composition $\mathrm{M}(\mathrm{P})$ of all the grid nodes is calculated with the source based material composition calculation formulas of Eq.1 to Eq.5 and represented in an accurate two-dimension vector $\left[w_{s}, w_{e}\right]$.

(3)Color mapping: After the above steps, with the color mapping technology, the calculated $w_{s}$ and $w_{e}$, as scalar values of the specific color channel, are mapped into system color. For the cladding part, the interface material is composed of the substrate and clad materials. Let blue and red represent the substrate and clad materials respectively, to a given grid node, if $\mathrm{M}(\mathrm{P})=[0.6,0.4]$, the responding systemic color is [0.6,0,0.4].

(4)Grid rendering: Grid rendering follows the material calculation and color mapping. Color mixture (linear interpolation) method[8] in the OpenGL rendering engine is used to render the triangular girds as shown in Fig.3. In the gird with nodes of $\mathrm{P}_{1}, \mathrm{P}_{2}$ and $\mathrm{P}_{3}$, to an arbitrary point $\mathrm{P}_{12-23}$, $\mathrm{P}_{1-2}$ and $\mathrm{P}_{1-3}$ are the intersection points of the scanning line through $\mathrm{P}_{12-23}$ and the grid boundaries. The $\mathrm{P}_{12-23}$ color is calculated by colors of $\mathrm{P}_{1-2}$ and $\mathrm{P}_{1-3}$ with linear interpolation method, the colors of $\mathrm{P}_{1-2}$ and $\mathrm{P}_{1-3}$ are obtained by those of $\mathrm{P}_{1}, \mathrm{P}_{2}$ and $\mathrm{P}_{1}, \mathrm{P}_{3}$ respectively.

After all the grid surfaces are rendered, the interface layer rendering will be completed.

Assistant Modeling of the Interface Layer. This modeling process mainly includes union operation, unit refining, unit material query and alteration.

If the interface layer is not modeled at one time, and all the interface layers modeled in different times have

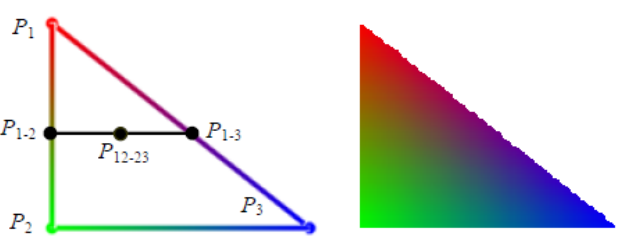

Fig.3 Rendering the grid element intersection parts, union operation may be applied to keep these layers as a whole one. Unit refining means to refine some discretized units to make local material representation with more accurate if necessary. Unit material query and alteration make it possible to query material information of each voxel unit and modify it if necessary.

Clad Layer Modeling. The clad layer modeling includes geometry modeling, material information addition and visualization. Geometry modeling process is similar to that of the interface layer, the material information addition and visualization methods are same to that of the substrate layer.

Glue and Assistant Modeling. In order to finish the model of the whole cladding part, processes of glue and assistant modeling are necessary.

Glue. A cladding part is made up of three layers of the substrate, interface and clad, after these layers are modeled, they need to be glued together to form a whole part.

Glue process includes geometric glue and bonding strength addition. Geometric glue means to glue the adjacent surfaces between the layers of the substrate, interface and clad together to make them become a whole one in geometry. As the cladding part is fabricated through physical or chemical method in practice, there exists bonding strength in the adjacent surfaces between layers. The bonding strength is added to the adjacent surfaces as their attributions in this paper.

Assistant Modeling. In the paper, the named assistant modeling of the cladding part includes operations of difference, rounding or chamfering of the solid containing the interface. Geometric Boolean operations and material composition updating occur within all of these operations.

Geometric operations of cladding parts are same to homogeneous ones. Material updating process is as follows. After geometric Boolean operations, the material attribution of the new generated surface is determined by that of the cladding part. The material and color attributions of the new generated surfaces in the clad and substrate will not change, while elements in the interface layer affected by geometric operations will be re-discreted and modified to keep the voxel unit continuity. The new surfaces will be re-rendered with the interface rendering method.

\section{Modeling System Prototype for the Cladding Part}


Based on the hierarchical modeling method, using UIDesigner tools and existing modeling functions of the software SINOVATION, the modeling system prototype for the cladding part is designed. Its main interface is shown in Fig.4. Tools for modeling the cladding part in the $\mathrm{T}$ area include substrate material definition, clad material definition, interface layer modeling, clad layer modeling, glue, heterogeneous solid difference operation, rounding and chamfering, and interface layer union operation. The interface layer modeling includes its basic and assistant modeling (unit refining, unit material query and alteration).

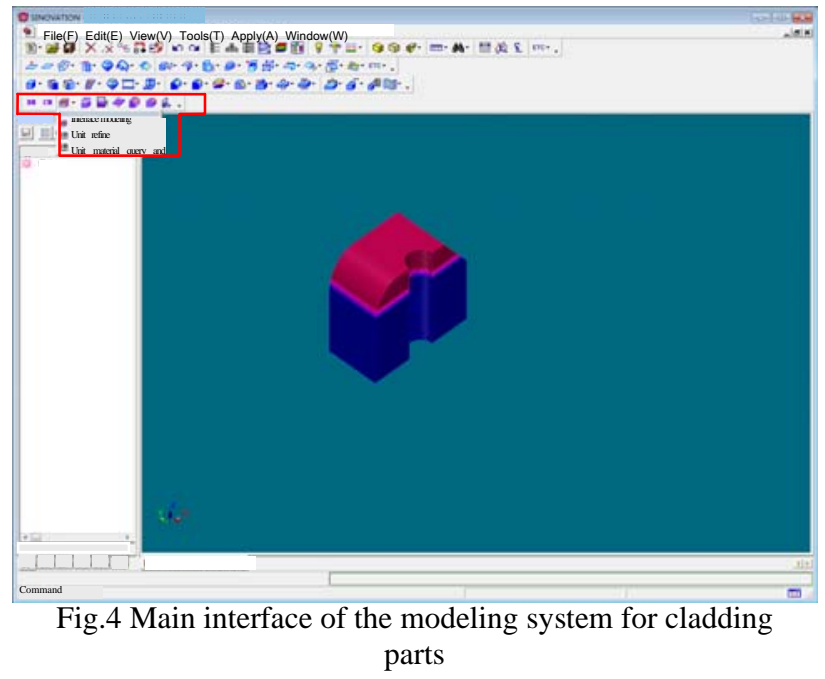

\section{Summary}

A hierarchical modeling method for cladding parts is proposed, and the modeling system prototype is designed based on SINOVATION.

In the method, the substrate, interface and clad are modeled and their material attribution is added respectively to avoid complicated heterogeneous material Boolean operations. The interface is modeled based on the substrate and the clad is modeled based on the interface, which makes their sizes exist relevance to modify the model conveniently. Bonding strength is taken into consideration to benefit the following CAE integration.

\section{Acknowledgements}

This project is supported by International Cooperation Project from Chinese Ministry of Science and Technology (2011DFB11490), Chinese National High Technology Research and Development Program (863 Program, 2012AA040903) and Shandong Province Outstanding Young Scientist Research Award Fund (BS2009ZZ012).

\section{References}

[1] Yang Junru, Jia Qiaohui, Chen Gongling, etal: Coal Mine Machinery, Vol.31 (2010), p19

[2] V Kumar, D Burns, Dutta, etal: Computer-Aided Design, Vol.31 (1999), p541

[3] Pasko A, Adzhiev, Schmitt B, etal: Graphical Models, Vol.63(2001), p 413

[4] Xu Rufeng, Chen Zhitong and Chen Wuyi: Computer Integrated Manufacturing Systems, Vol.17 (2011), p 95

[5] Li Shuping, Yan Kun, Li Huan and Zhang Guangyan: Journal of Engineering Graphics, No.1 (2006), p 116

[6] Liao Ping: Computer Simulation, Vol.26 (2009), p176

[7] Zhan Haisheng, Li Guangxin and Ma Zhixin: Geometry modeling technology and system development base on ACIS (Tsinghua University Press, Beijing 2002)

[8] Kou Xinyu: Computer-aided design of heterogeneous objects (The University of Hong Kong, Hong Kong 2005) 